\title{
The timing of reperfusion therapy
}

\author{
Bernard J Gersh
}

A fundamental tenet of reperfusion therapy is that 'time is muscle', since ischemia leading to necrosis is progressive. Regardless of whether treatment is pharmacologic or primary percutaneous coronary intervention (PCl), evidence is accumulating that delays at each stage in the process are associated with adverse outcomes. Indeed, the ability to deliver therapy expeditiously is generally accepted as a surrogate for quality of care.

Although the preferred strategy in institutions with the requisite facilities and expertise is primary $\mathrm{PCl}$, several other approaches can be used in hospitals without catheterization facilities. The treatment options are critically dependent on the duration of symptoms and the time expected to elapse before achievement of reperfusion. The relationship between duration of ischemia, mortality reduction and the extent of myocardial salvage shows a striking benefit if the procedure is performed within the first 2-3h after onset of ischemia. This finding emphasizes the narrow but 'golden' window of opportunity. After this time, the relationship curve flattens and, although a mortality benefit can still be shown, its magnitude decreases over time. This relationship might be affected by several variables, such as myocardial oxygen demand, collaterals and perhaps ischemic preconditioning. The slope of the curve can be used to provide a rationale for determining optimum reperfusion strategies in community hospitals without primary $\mathrm{PCl}$ capability.

During the initial $2 \mathrm{~h}$ of ischemia, time to treatment is pivotal, and urgent administration of intravenous fibrinolytic drugs is the primary therapy in hospitals without $\mathrm{PCl}$ facilities. Subsequent transfer of the patient to a referral hospital for so-called rescue angioplasty or angiography is appropriate, but whether angiography should be ischemia-driven or routine, and the timing of the routine procedure, is the subject of ongoing trials.

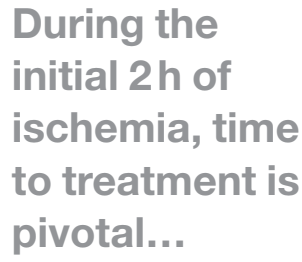

By contrast, as the time from symptom onset increases and the curve flattens, the speed of therapy becomes less of a factor, and the opening of the infarct-related artery becomes the priority. To achieve this goal a mechanical approach is preferable; thrombi are thought to become more resistant to fibrinolysis over time, whereas the efficacy of $\mathrm{PCl}$ is sustained. In patients presenting later, therefore, a strong case can be made for direct transfer for immediate primary $\mathrm{PCl}$ without thrombolytic pretreatment. Facilitated primary $\mathrm{PCl}$, after pretreatment with thrombolytics, seems a promising and logical therapy, particularly if transport times to $\mathrm{PCl}$ facilities are lengthy. Preliminary results from one large trial are, however, discouraging, and further rigorous scrutiny of randomized, controlled trials is needed. As is the case with all procedures, new strategies must be bolstered by clinical judgment. Assessment should include bleeding risk, hemodynamic stability and a realistic estimate of transport delays. In many communities, the strategy of prehospitalization thrombolytic therapy might be the best approach.

After about three decades of reperfusion therapy, the landscape of treatment for STelevation myocardial infarction has changed dramatically and irrevocably. Initial and understandable enthusiasm for the logic of reperfusion of the infarct-related artery has been confirmed by clinical trials. Many pathophysiologic and therapeutic issues have been answered, although the attainment of optimum myocardial perfusion and preservation of microvascular function has been disappointing. The other major issues in reperfusion therapy are logistical; we know what to do, the question is how best to implement therapy. The goals, however, are unambiguous - to treat as many eligible patients as quickly as possible. 